\title{
The Stabilizing Function of Superficial Shoulder Muscles Changes Between Single-Plane Elevation and Reaching Tasks
}

\author{
Diederik J. D. Ameln, Edward K. Chadwick, Dimitra Blana and Alessio Murgia*, Member, IEEE
}

\begin{abstract}
Objective: The goal of the current study was to determine whether and how much the stabilizing role of the shoulder muscles changes as a function of humeral elevation and the plane of elevation. Methods: A musculoskeletal model, comprising a personalized scapulohumeral rhythm, was used to calculate the ratio of shear over compressive force (stability ratio) of three rotator cuff muscles (supraspinatus, infraspinatus, subscapularis) and three superficial shoulder muscles (middle deltoid, clavicular part of pectoralis major, latissimus dorsi) during abduction, flexion and reaching movements in ten healthy adults. Results: The range of the stability ratios was $[ \pm 0.5]$ for the rotator cuff muscles compared to $[+5,-2]$ for the superficial shoulder muscles. In the superior-inferior direction, the stability ratios of all muscles changed with humeral elevation and for infraspinatus, subscapularis, latissimus dorsi and deltoid also with the plane of elevation. In the anterior-posterior direction, the stability ratios of all muscles changed with humeral elevation, except for the deltoid, and with the plane of elevation, except for the supraspinatus, with interaction effects in all muscles. Conclusion: The rotator cuff muscles provide greater compression than shear forces during all tasks. The stabilizing function of the superficial shoulder muscles examined in this study varies during tasks. Significance: The findings can be used to predict in which movements the shoulder joint becomes more unstable and can be applied to understand how shear and compressive forces change in populations with abnormal shoulder motion.
\end{abstract}

Index Terms-Glenohumeral stability, Musculoskeletal modeling, Upper limb biomechanics.

\section{INTRODUCTION}

$\mathbf{T}$ HE relative movement between upper arm and thorax in the human shoulder is accomplished through the combined degrees of freedom of the joints forming the shoulder girdle and the glenohumeral joint, and by the gliding of the scapula over the scapulothoracic plane [1]. A disadvantage of this complex mechanism is that it causes the shoulder and the glenohumeral joint in particular to be inherently unstable [2].

This unstable nature, combined with the fact that the shoulder joint is used intensively in a wide range of daily life tasks, makes the joint susceptible to overuse injuries such as rotator cuff tears and arthritis [2], [3], [4]. Epidemiological estimates,

Manuscript received September 27, 2017; revised March 15, 2018 and May 20, 2018; accepted June 15, 2018. Date of publication ???; date of current version June 22, 2018.

D. J. D. Ameln is with University of Groningen, The Netherlands. E. K. J. Chadwick and D. Blana are with Keele University, UK. *A. Murgia is with University of Groningen, University Medical Center Groningen, Center for Human Movement Sciences, The Netherlands. (correspondence e-mail: a.murgia@umcg.nl). accounting shoulder injuries for as much as $16 \%$ of all musculoskeletal complaints, reflect this vulnerability [4]. Mobility of the humerus is achieved by the action of the superficial shoulder muscles and is extended through scapular motion [5]. The scapula rotates laterally along with the humerus in an approximate $2: 1$ ratio starting at about $30^{\circ}$ of humeral elevation in the coronal plane, which allows orienting the glenohumeral joint surface in the direction of movement [1], [6]. The combined rotations of humerus, clavicle and scapula during humeral elevation follow a regular pattern within and between individuals [7], known as the scapulohumeral rhythm [8]. As a result of these rotations the muscles' lines of action and the forces across the joint change during arm elevation.

Estimation of these forces is important to understand how the stability of the glenohumeral joint is affected by the type of movement. In the non-pathological shoulder, active stability is achieved by the compressive forces of the rotator cuff muscles, which act to mantain the head of the humerus firmly in contact with the glenoid cavity [9]. The small moment arms and short fibre lengths of the rotator cuff muscles enable them to produce a rapid force over a small change in muscle length [10]. Although these properties are evidence of a stabilizing role, the functional role of individual rotator cuff muscles during movement can be more difficult to establish [11], [12], [13] and should always be seen in the context of the kinematics of the movement [11], [14]. Moreover, several superficial shoulder muscles such as deltoid, latissimus dorsi and pectoralis exert shear and compressive forces, which can also affect joint stability [15] and might help preserve function in the event of rotator cuff tears [16]. A deeper understanding of the rotator cuff and superficial muscles' functional role can therefore help to further comprehend the possible causes of pathological shoulder mobility.

In order to approach this problem it is useful to study the individual muscles' contribution to mobility and joint stability. Glenohumeral joint stability can be described through the resultant of the compressive and shear muscle forces. The stability ratio has been defined as the ratio between the shear and compressive components of the muscle force with respect to the joint surface and can be seen as a measure of the stabilizing potential of the muscle [2], [17]. Stability ratios of the rotator cuff and superficial shoulder muscles have been reported in cadaveric studies during movements imposed on the body segments by the experimenters [17], [18]. However 
these cadaver studies have limited validity and application to in vivo kinematics. Additionally electromyographic studies have examined phasic activity of the shoulder muscles [11], [12] but cannot shed light on the changes in stability ratio during a movement.

Musculoskeletal models, on the other hand, allow investigating movements in vivo while taking muscle activity into account [19], [20], [21]. In this way it has been shown that supraspinatus, infraspinatus and subscapularis contributed little to the glenohumeral abduction torque and presented low stability ratios during humeral abduction in the scapular plane [22]. However complex movements have not been considered yet and it is therefore not clear whether and how the rotator cuff and superficial muscles' functional roles depend on the plane of elevation or how their stabilizing potential varies with increasing reaching distance. The aim of the current study was to determine whether and how much the stabilizing role of the shoulder muscles changes depending on humeral elevation and the plane of elevation, represented by the type of task. A musculoskeletal model was used to calculate the stability ratios of the main rotator cuff muscles and selected superficial shoulder muscles during abduction, flexion and reaching. It was hypothesized that the stability ratio of the rotator cuff muscles would remain unaffected by changes in humeral elevation and plane of elevation to provide glenohumeral stability during the entire range of motion. On the other hand the stability ratio of the superficial shoulder muscles was hypothesized to vary as a function of humeral elevation due to the mobilizing forces exerted by these muscles around the joint but to remain unaffected by the plane of elevation.

\section{Methods}

\section{A. Participants}

Healthy participants volunteered for the study $(\mathrm{N}=10$; mean age $22.5 \pm 2.1$ ys, mass $67.1 \pm 7.7 \mathrm{~kg}$, height $176.3 \pm 11.6 \mathrm{~cm}$ ). Exclusion criteria were left-hand dominance and current or past shoulder injuries. All participants were informed about the procedures of the experiment and signed an informed consent. The study was approved by the Ethics Board of the Center of Human Movement Science (University Medical Center Groningen, the Netherlands, reference: ECB/2016.01.29_1) and the protocol was in accordance with the declaration of Helsinki.

\section{B. Procedure}

The movement trials consisted of a block of three planar elevation movements and a block of three reaching tasks per participant. The order of the blocks and the trials within the blocks were randomized to control for possible muscle fatigue. Participants remained seated on a stool without back support for the full duration of the measurements. During the planar elevation tasks participants were instructed to elevate the dominant arm three times consecutively up to the full range of motion in the coronal (abduction), scapular and sagittal (forward flexion) plane while keeping the trunk upright, their elbow extended and moving at comfortable speed. During the reaching tasks, participants sat in front of a table touching a
TABLE I

LOCATIONS OF MOTION CAPTURE MARKERS

\begin{tabular}{ll}
\hline \hline Segment & Location on body \\
\hline Thorax & $7^{\text {th }}$ cervical spinous process \\
& $10^{\text {th }}$ thoracic spinous process \\
Xiphoid process of the sternum \\
Clavicle & $\begin{array}{l}\text { Jugular notch of clavicle } \\
\text { Acromioclavicular joint }\end{array}$ \\
Humerus & $\begin{array}{l}\text { Lateral epicondyle of the elbow } \\
\text { Medial epicondyle of the elbow }\end{array}$ \\
Radius & Radial styloid process \\
Ulna & Ulnar styloid process \\
Hand & Head of $3^{\text {rd }}$ metacarpal \\
\hline \hline
\end{tabular}

start position located in the sagittal plane, with the arm vertical and the elbow at $90^{\circ}$. They were then instructed to reach from this position to a target three times at comfortable speed. The target's vertical position was located at one shoulder height. The target's horizontal position was located either in the middle, in front of the participant's dominant shoulder using the acromion as reference, or on the ipsilateral or contralateral side at one shoulder width from the middle. Shoulder width was defined as the distance between the left and right acromion.

\section{Instrumentation and Data Collection}

EMG activity was collected at $1500 \mathrm{~Hz}$ using wireless surface electrodes (Trigno, Delsys Inc., Natick, MS, USA). EMG electrodes were placed on the upper trapezius, lower trapezius, serratus anterior and the middle and anterior part of the deltoid muscles because of their functional role during upper arm elevation movements. Maximum voluntary isometric contractions (MVC) were measured beforehand based on recommendations for the normalization procedures of shoulder muscle activity [23]. Three MVC trials, each lasting 5 seconds, were measured with a period of rest in between. Body segment positions were collected at $100 \mathrm{~Hz}$ using a 3 position sensor motion capture system (Optotrak 3020, Northern Digital Inc., Waterloo, ON, Canada). Ten infrared light emitting markers were placed on superficial bony landmarks of the upper limb and thorax segments. Three marker clusters, consisting of 3 markers each, were additionally placed on the lateral side of the upper arm and on the dorsal side of the lower arm to reconstruct eventual missing wrist and elbow markers. The marker locations are shown in Table I.

\section{Data Treatment}

EMG signals were filtered with a 4th order bandpass Butterworth filter with cut-off frequencies of $20-450 \mathrm{~Hz}$. The signals were rectified and smoothed using a root-mean-square filter with a window of $71 \mathrm{~ms}$. EMGs were normalized using the maximum value derived from all the isometric MVC trials for a given muscle.

Cartesian coordinates of the infrared markers were reconstructed using linear interpolation whenever gaps were present and used to drive the musculoskeletal model. 


\section{E. Musculoskeletal Model}

The OpenSim musculoskeletal modeling software [24], [25] was used to run a model of the shoulder and the upper arm. The modified Dynamic Arm Simulator model (DAS3) was used [26], which is based on the dataset of the Delft Shoulder and Elbow Model (DSEM) [27] and consists of 7 bone segments and 138 muscle elements (Figure 1). Glenoid version and inclination were $12^{\circ}$ (retroversion) and $4^{\circ}$ (superior) respectively. The DAS3 model was scaled in OpenSim to the body dimensions of the participants using motion capture data and was modified to incorporate a personalized scapulohumeral rhythm derived from measurements carried out in this study.

The rotations of clavicle and scapula relative to the thorax were based on individual measurements of the acromion and of scapular landmarks using a scapula locator [28]. Individualized regression equations were then derived to estimate clavicle and scapular orientation from the humeral elevation angle. The scapular locator has been shown to be valid [29] and to have good inter- and intra-observer reliability [30] in the measurement of scapulohumeral kinematics. In the locator, three adjustable plastic pins were moved to coincide with the inferior angle, trigonum spinae and the acromial angle of the scapula of each participant. Following this, static poses of the pin positions relative to the thorax coordinate system were recorded twice at 12 different elevation angles in the coronal plane, equally spaced between $15^{\circ}$ and $180^{\circ}$, measured using a goniometer. Finally the $Y^{\prime} X^{\prime} Z$ ' Euler rotations of the clavicle and scapula, and $Y^{\prime} X^{\prime} Y^{\prime}$ Euler rotations of the humerus relative to the thorax coordinate system were calculated following ISB recommendations, where the $\mathrm{Y}$ axis points upwards, the $\mathrm{X}$ axis lies in the posterior-anterior direction and the $\mathrm{Z}$ axis points to the right side [31]. Rotations were then expressed as

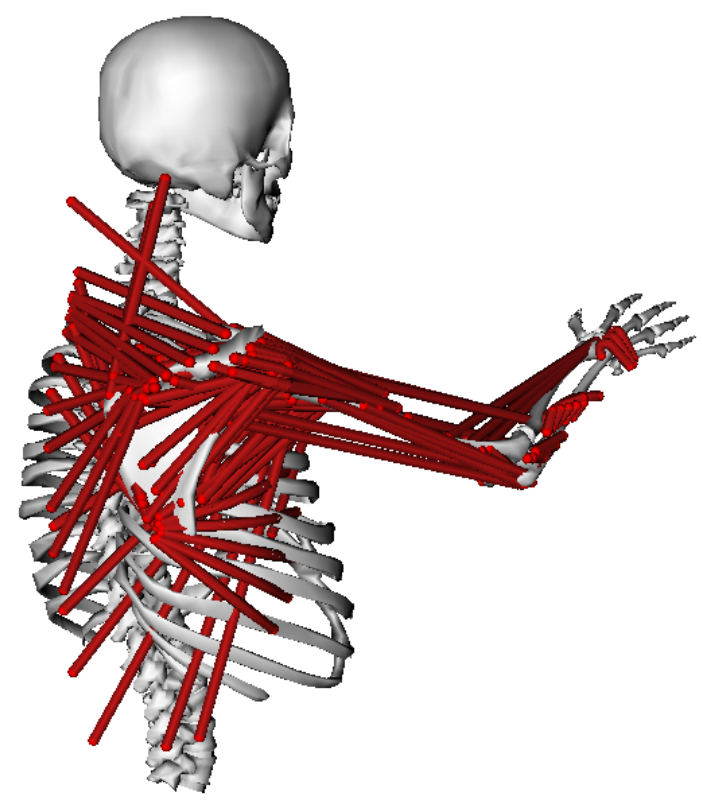

Fig. 1. Dorsolateral view of the geometry of the musculoskeletal model used in this study.
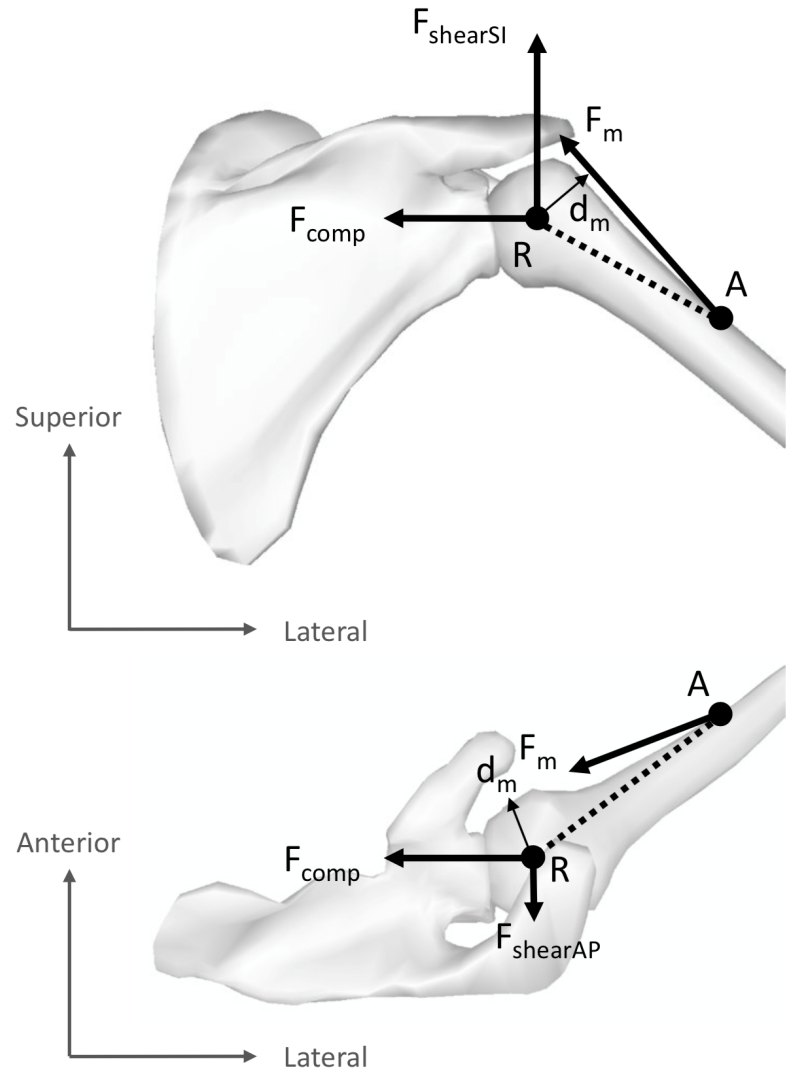

Fig. 2. The line of action and attachment site of a muscle can be used to calculate stability ratio and moment arm. $\mathrm{F}_{\mathrm{m}}$ : projection of muscle force; $\mathrm{F}_{\text {shear }}$ : shear force component; $\mathrm{F}_{\text {comp }}$ : compressive force component; $\mathrm{R}$ : center of rotation; A: muscle attachment site; $\mathrm{d}_{\mathrm{m}}$ : moment arm of muscle force.

a second order polynomial function of the humeral elevation angle for each participant. A regression-based shoulder rhythm approach based on measurements from a scapular locator has been found to be a valid representation of bone orientations [32]. Moreover, personalized scapular kinematic models have also been shown to be valid at estimating glenohumeral forces during shoulder abduction and flexion movements [33].

Activations of individual muscles were subsequently estimated in OpenSim using a static optimization process. The muscles individual force components and the resultant glenohumeral joint force were calculated using the OpenSim application programming interface in MATLAB (Mathworks Inc., Natick, MA, USA). There was no stability constraint on the glenohumeral joint force. The cost function used was the sum of the squared muscle activation levels [34]:

$$
J=\sum_{m=1}^{n}\left(a_{m}\right)^{2}
$$

where $n$ is the number of muscles and $a_{m}$ the activation of muscle $m$ at a discrete time step.

\section{F. Analysis}

The scapulohumeral model used in this study was validated by comparing the estimated muscle activations to the electromyographic (EMG) activities of shoulder muscles 
measured with surface electrodes. Cross-correlation between estimated and measured EMG activities [35] was performed beforehand to correct for the electromechanical delay. The muscle activation signals were compared by calculating the normalized cross-correlation coefficients at zero lag, between the model estimates, and the measured EMG activities for elevations up to $90^{\circ}$ and above $90^{\circ}$ [36], [37], [38]. Correlation coefficients were transformed to Fisher's $\mathrm{z}$ before averaging across participants to reduce bias [39]. Correlations higher than 0.5 were considered to indicate agreement between measurements and predictions [40]. The root mean square error between estimated and measured EMG activities was used to quantify the absolute error. Additionally, the resultant of the glenohumeral joint force estimate from the model was validated by comparing it with the values from the literature as a function of the humeral elevation angle.

Stability was quantified by means of the stability ratio, which was defined as the ratio between the shear and compressive components of the muscle force with respect to the joint surface [2], [17]. A stability ratio between 1 and -1 indicates that the compressive force component of the muscle is greater than the shear component, whereas a stability ratio greater than 1 or smaller than -1 indicates that the muscle has a greater potential to destabilize the joint. The stability ratios were calculated as a function of the upper arm elevation angle [17]. Stability ratios for each muscle were calculated for the middle deltoid, clavicular part of the pectoralis major, latissimus dorsi and for the rotator cuff muscles supraspinatus, infraspinatus and subscapularis. Teres minor was not included in the analysis because its line of action is almost exactly aligned with that of the infraspinatus muscle [18]. The vector pointing from the distal to the proximal attachment site of each muscle element identified the muscle's line of action. The vector was expressed in the coordinate system of the glenohumeral joint to compute one compressive and two shear components of the muscle force. Figure 2 shows the decomposition of muscle force in shear and compressive components in the superior-inferior (SI) and anterior-posterior (AP) directions. The stability ratios along the SI and AP directions were then calculated by taking the ratio between the shear and compressive force components:

$$
S R_{S I}=\frac{F_{\text {shear } S I}}{\left|F_{\text {comp }}\right|}, S R_{A P}=\frac{F_{\text {shear } A P}}{\left|F_{\text {comp }}\right|} .
$$

\section{G. Statistics}

To test the hypothesis concerning the rotator cuff and superficial shoulder muscles, i.e. whether their stability ratios were affected by the amount of humeral elevation and plane of elevation, a multi-factorial repeated-measures ANOVA was carried out using the stability ratios as dependent variable for the supraspinatus, infraspinatus, subscapularis, middle deltoid, pectoralis major and latissimus dorsi muscles. Within-subjects factors were the humeral elevation, with 6 levels between $15-90^{\circ}$ spaced at $15^{\circ}$ intervals, and the type of movement (associated to the plane of elevation): abduction, abduction in the scapular plane, forward flexion and reaching to the ipsilateral, front and contralateral side. The ANOVA (IBM SPSS Statistics version 23, IBM Corporation, Armonk, NY,

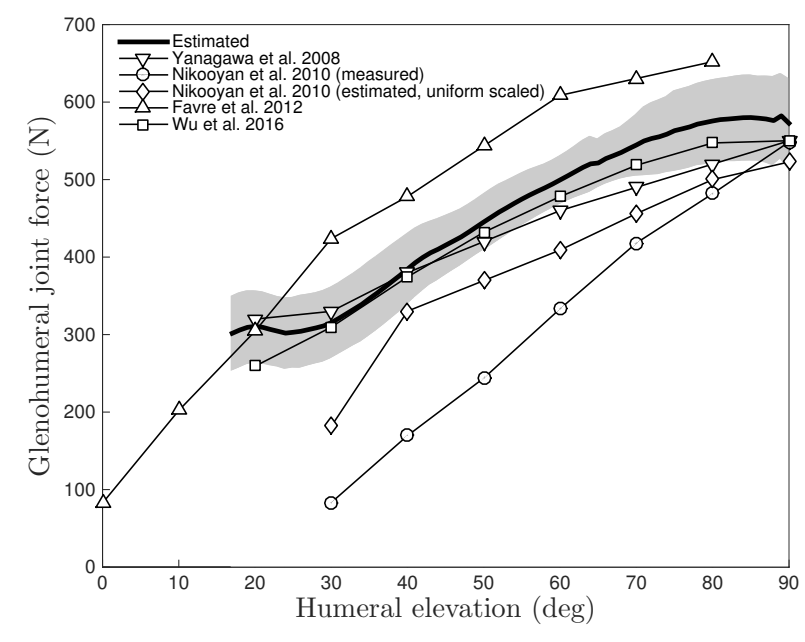

Fig. 3. Glenohumeral joint force estimated from the current model (thick black line represents the population mean, the grey band represents \pm 1 SD) and from literature data [38], [41], [21], [22] as a function of humeral elevation during abduction.

USA) was performed in both SI and AP directions with $\alpha=0.05$. Effect size was reported as partial $\eta$ squared $\left(\eta_{p}^{2}\right)$. Follow-up planned contrasts were done for the superficial muscles afterwards.

\section{RESULTS}

\section{A. Muscle Activity and Glenohumeral Joint Force}

High correlations $(>0.5)$ were found between modelestimated and measured EMG activities in the reaching tasks for all muscles except for the lower trapezius (Table II). During the planar elevation movements model estimates also correlated highly with measured EMG up to $90^{\circ}$ of humeral elevation. Similar results were found for the absolute error between model estimates and measured activity, which was higher for the elevation movements than for the reaching tasks (Table III). The resultant of the glenohumeral joint force estimate was consistent in magnitude and pattern with that of other studies as seen in Figure 3.

\section{B. Stability Ratio}

The values of the stability ratios of the rotator cuff muscles across the six tasks are shown in Figure 4. Along the SI direction, the infraspinatus, subscapularis and supraspinatus muscles provided greater stability throughout the different tasks, as shown by the low stability ratios in the range $[ \pm 0.5]$. As illustrated by the sign of the stability ratios, shear force components of infraspinatus and supraspinatus were directed anteriorly whereas the shear force of the subscapularis was directed posteriorly. In the SI direction, the shear force component of the supraspinatus was directed superiorly whereas the shear force components of infraspinatus and subscapularis were both directed inferiorly.

The stability ratios of the infraspinatus muscle in the SI and AP directions differed significantly across elevations (SI: $F(1.2,5.8)=54.4, \eta_{p}^{2}=0.92$; AP: $F(1.2,6)=39.6, \eta_{p}^{2}=$ 0.88 ; all $p \leq 0.001$ ) and movements (SI: $F(5,25)=21.3$, 
TABLE II

CROSS-CORRELATION COEFFICIENTS BETWEEN MEASURED EMGS AND MODEL ESTIMATES, CORRECTED FOR ELECTROMECHANICAL DELAY AND TRANSFORMED TO FISHER'S Z. EACH VALUE REPRESENTS THE MEAN ACROSS PARTICIPANTS $(\mathrm{N}=10)$.

\begin{tabular}{|c|c|c|c|c|c|c|c|c|c|c|}
\hline & \multicolumn{2}{|c|}{ UT } & \multicolumn{2}{|c|}{ LT } & \multicolumn{2}{|c|}{ SA } & \multicolumn{2}{|c|}{ MD } & \multicolumn{2}{|c|}{$\mathrm{AD}$} \\
\hline & $\leq 90^{\circ}$ & $>90^{\circ}$ & $\leq 90^{\circ}$ & $>90^{\circ}$ & $\leq 90^{\circ}$ & $>90^{\circ}$ & $\leq 90^{\circ}$ & $>90^{\circ}$ & $\leq 90^{\circ}$ & $>90^{\circ}$ \\
\hline Abduction & 0.589 & -0.454 & 0.230 & -0.262 & 0.642 & -0.592 & 0.798 & -0.456 & 0.853 & -0.649 \\
\hline Scap Elev & 0.545 & -0.299 & 0.334 & -0.162 & 0.639 & -0.538 & 0.826 & -0.584 & 0.840 & -0.554 \\
\hline Forw Flex & 0.608 & -0.195 & 0.407 & -0.174 & 0.652 & -0.594 & 0.822 & -0.614 & 0.856 & -0.473 \\
\hline Reach C & 0.491 & & 0.205 & & 0.691 & & 0.916 & & 0.894 & \\
\hline Reach F & 0.523 & & 0.147 & & 0.719 & & 0.905 & & 0.890 & \\
\hline Reach I & 0.654 & & 0.021 & & 0.709 & & 0.922 & & 0.872 & \\
\hline
\end{tabular}

Conditions: Abduction: regular abduction; Scap Elev: scapular plane elevation; Forw Flex: forward flexion; Reach C: contra-lateral reaching; Reach F: reaching towards the front; Reach I: Ipsilateral reaching. Muscles: UT: upper trapezius; LT: lower trapezius; SA: serratus anterior; MD: middle deltoid; $\mathrm{AD}$ : anterior deltoid.

TABLE III

Root MeAn Square ERror (as FRACTION OF MVC; 1 REPRESENTS 100\% MVC) BETWEEN MEASURED EMGS AND ESTIMATED MUSCLE ACTIVITY. EACH VALUE REPRESENTS THE MEAN ACROSS PARTICIPANTS $(\mathrm{N}=10)$.

\begin{tabular}{|c|c|c|c|c|c|c|c|c|c|c|}
\hline & \multicolumn{2}{|c|}{ UT } & \multicolumn{2}{|c|}{ LT } & \multicolumn{2}{|c|}{ SA } & \multicolumn{2}{|c|}{ MD } & \multicolumn{2}{|c|}{$\mathrm{AD}$} \\
\hline & $\leq 90^{\circ}$ & $>90^{\circ}$ & $\leq 90^{\circ}$ & $>90^{\circ}$ & $\leq 90^{\circ}$ & $>90^{\circ}$ & $\leq 90^{\circ}$ & $>90^{\circ}$ & $\leq 90^{\circ}$ & $>90^{\circ}$ \\
\hline Abduction & 0.049 & 0.166 & 0.045 & 0.209 & 0.045 & 0.161 & 0.050 & 0.210 & 0.030 & 0.170 \\
\hline Scap Elev & 0.047 & 0.151 & 0.045 & 0.186 & 0.055 & 0.155 & 0.048 & 0.195 & 0.045 & 0.175 \\
\hline Forw Flex & 0.041 & 0.148 & 0.043 & 0.192 & 0.047 & 0.170 & 0.046 & 0.177 & 0.059 & 0.185 \\
\hline Reach C & 0.039 & & 0.013 & & 0.044 & & 0.032 & & 0.061 & \\
\hline Reach F & 0.040 & & 0.025 & & 0.044 & & 0.033 & & 0.045 & \\
\hline Reach I & 0.030 & & 0.028 & & 0.044 & & 0.031 & & 0.034 & \\
\hline
\end{tabular}

Conditions: Abduction: regular abduction; Scap Elev: scapular plane elevation; Forw Flex: forward flexion; Reach C: contra-lateral reaching; Reach F: reaching towards the front; Reach I: Ipsilateral reaching. Muscles: UT: upper trapezius; LT: lower trapezius; SA: serratus anterior; MD: middle deltoid; AD: anterior deltoid.

$\eta_{p}^{2}=0.81 ; p<0.001 ;$ AP: $F(1.2,6)=7.3, \eta_{p}^{2}=0.59$; $p=0.03)$ with a significant interaction effect between movement and elevation (SI: $F(3.1,15.6)=15.2, \eta_{p}^{2}=0.75$; AP: $F(2,10)=23.9, \eta_{p}^{2}=0.83$; all $\left.p<0.001\right)$. Similarly for the subscapularis muscle in both SI and AP directions main effects of elevation (SI: $F(1.2,5.9)=70.9, \eta_{p}^{2}=0.93$; AP: $F(1.2,6)=57.8, \eta_{p}^{2}=0.92$; all $\left.p<0.001\right)$, movement (SI: $F(5,25)=15.2, \eta_{p}^{2}=0.75 ;$ AP: $F(1.4,7)=$ $12.9, \eta_{p}^{2}=0.72$; all $p<0.01$ ) and relative interaction (SI: $F(3.5,17.5)=9.0, \eta_{p}^{2}=0.64$; AP: $F(3.4,16.8)=5.1$, $\eta_{p}^{2}=0.5$; all $\left.p<0.01\right)$ were found. For the supraspinatus muscle a significant main effect was only found for elevation in both SI and AP directions (SI: $F(1.1,5.6)=74.5$; AP: $F(1.2,6)=77.2$; all $\eta_{p}^{2}=0.94$; all $\left.p<0.001\right)$, with an interaction effect between movement and elevation in the AP direction only $\left(F(2.3,11.4)=21.6, \eta_{p}^{2}=0.81 ; p<0.001\right)$.

In contrast to the rotator cuff muscles the stability ratios of the superficial muscles had a greater range $[+5,-2]$. In the SI direction in particular, greater stability ratios were seen at lower values of humeral elevation and decreased with increasing elevation (Figure 4). The middle deltoid and pectoralis major showed a (positive) superior shear component at lower levels of humeral elevation, in contrast to the (negative) inferior shear component of the latissimus dorsi. The stability ratios of the middle deltoid differed significantly across elevations in the SI direction only $\left(F(1.1,5.6)=43.0, \eta_{p}^{2}=0.90\right.$; $p=0.001)$ and across movements in the SI and AP directions (SI: $F(1.3,6.6)=23.0, \eta_{p}^{2}=0.82$; AP: $F(1.4,7)=32.2$, $\eta_{p}^{2}=0.87$; all $\left.p<0.01\right)$ with a significant interaction effect between movement and elevation in both SI and AP directions (SI: $F(1.9,9.5)=8.6, \eta_{p}^{2}=0.63$; AP: $F(2.8,14.2)=17.3$, $\eta_{p}^{2}=0.78$; all $p<0.01$ ). For the latissimus dorsi muscle the stability ratios in both SI and AP directions differed between elevations (SI: $F(1.1,5.5)=260, \eta_{p}^{2}=0.98$; AP: $F(1.2,6)=9.2, \eta_{p}^{2}=0.65$; all $\left.p<0.02\right)$, movements (SI: $F(5,25)=14.5, \eta_{p}^{2}=0.74$; AP: $F(1.8,9)=40.9$, $\eta_{p}^{2}=0.89$; all $\left.p<0.001\right)$ and there was an interaction effect beween movement and elevation (SI: $F(3.8,19.2)=8.4$, $\eta_{p}^{2}=0.63 ;$ AP: $F(2.5,12.3)=14.9, \eta_{p}^{2}=0.75$; all $p<0.001)$. For the pectoralis muscle a significant main effect was found for elevation in the SI and AP directions (SI: $F(1.3,6.5)=63.0, \eta_{p}^{2}=0.93$; AP: $F(1.2,6.2)=43.9$, $\eta_{p}^{2}=0.90$; all $\left.p<0.001\right)$ and for movement in the AP direction only $\left(F(1.4,6.9)=32.3, \eta_{p}^{2}=0.87 ; p=0.001\right)$ with an interaction effect between movement and elevation in the AP direction only $\left(F(3.2,15.9)=12.2, \eta_{p}^{2}=0.71 ; p<0.001\right)$.

Contrasts revealed that for the middle deltoid in the SI and AP directions the stability ratios in abduction, flexion, contralateral and front reaching (SI only) were greater than the mean at different elevations vs. $90^{\circ}$ elevation (largest effect sizes: in the SI direction during front reaching at $60^{\circ} F(1,5)=87.1, \eta_{p}^{2}=0.95$ and in the AP direction during flexion at $15^{\circ} F(1,5)=58.1, \eta_{p}^{2}=0.92$ ). For the latissimus dorsi in the SI direction stability ratios were lower than the mean in all single plane elevation movements, contralateral and front reaching at different elevations vs. $90^{\circ}$ elevation whereas in the AP direction differences occurred in abduction, flexion and contralateral reaching only (largest 
effect sizes: in the SI direction during contralateral reaching at $15^{\circ} F(1,5)=54.8, \eta_{p}^{2}=0.91$ and in the AP direction during flexion at $\left.15^{\circ} F(1,5)=82.0, \eta_{p}^{2}=0.94\right)$. For the pectoralis in the SI direction a significant effect was only found for elevation, with all stability ratios at all elevations greater than ratios at $90^{\circ}$ elevation. In the AP direction stability ratios were greater than the mean at several elevations vs. $90^{\circ}$ in all single plane elevation movements, contralateral and front reaching (largest effect size: during flexion at $15^{\circ} F(1,5)=44.6$, $\left.\eta_{p}^{2}=0.9\right)$.

\section{Discussion}

The aim of the present study was to determine the stabilizing role of rotator cuff and selected superficial shoulder muscles as function of humeral elevation and the plane of humeral elevation. The stabilizing potential of the rotator cuff muscles remained high during the movements, with stability ratios less than 0.5 . In the supraspinatus the stability ratio was not affected by the plane of elevation. In the superficial shoulder muscles variability in stability ratios was larger within the movements as a function of humeral elevation and stability ratios tended to differ with the plane of elevation (between movements).

\section{A. Validation of the Musculoskeletal Model}

Muscle activities estimated by the model and measured with surface EMG were in agreement up to $90^{\circ}$ of humeral elevation. Moreover the resultant glenohumeral joint force was consistent with the values reported in the literature during humeral abduction [38], [41], [21], [22]. A discrepancy between estimated and measured surface EMGs was present for higher humeral elevation angles, as can be seen by the negative correlation values in Table II. This effect was caused by the model's underestimation of muscle activity at higher levels of humeral elevation and is consistent with the literature. A previous study using a similar musculoskeletal model to the current one found that estimated glenohumeral joint contact forces started deviating significantly from the measured forces for arm elevation angles above $90^{\circ}$ [38]. This underestimation originates from the way the model calculates muscle activities by optimally distributing the total load across the muscles. As elevation exceeds $90^{\circ}$, the net joint moment decreases [42], [19] and the model fails to take into account the co-contraction of antagonistic muscles that keeps the glenohumeral joint stable at these higher elevation angles. This results in estimated activation values that are lower than in reality. However, the stability ratios calculations take into account the lines of action of the muscles and thus although joint force estimation may not be correct above $90^{\circ}$ the forces are scaled by the same constant and the ratios of the force components are unaffected by the force magnitude.

\section{B. Glenohumeral Stability}

Low shear over compressive force, resulting in stability ratios less than unity, were found for the rotator cuff muscles throughout the movements confirming the stabilizing role of these muscles. Significant changes in the stability ratios, mainly for infraspinatus and subscapularis, were found as function of humeral elevation and plane of movement, which goes against the first part of the hypothesis. However, the stability ratios of the rotator cuff muscles remained confined within unity across the movements and the participants, indicating a constant stabilization effect on the glenohumeral joint. The findings during single plane humeral elevation are in line with a previous study using data from cadavers, where stability ratios of the rotator cuff muscles, averaged during the whole movement, were shown to have similar magnitudes in abduction and forward flexion [18]. Furthermore they are in line with the stability ratios found by others during forward flexion using a musculoskeletal model [22]. For the first time in this study, stability ratios during reaching tasks were provided and compared with other movements.

The infraspinatus and subscapularis muscles were found to contribute to joint stability by means of antagonistic action in the AP direction around the glenohumeral joint during both single plane elevation and reaching. In the SI direction the inferior destabilizing action of infraspinatus and subscapularis was counteracted by the superior action of the supraspinatus muscle. Whereas others have questioned the contribution of rotator cuff co-contraction to glenohumeral stability [43], in this study it was shown that the stability ratios of infraspinatus and subscapularis remained less than unity also during complex reaching tasks, which is evidence of a greater compressive force.

Results showed that the supraspinatus provides glenohumeral stability in all movement planes and during dynamic reaching tasks, as demonstrated by the small ratio of shear to compressive muscle force in both the superior and anterior direction. The primary role of the supraspinatus muscle during humeral elevation either as a glenohumeral stabilizer or as provider of the humeral elevation moment has been the object of extensive debate [19], [10], [44], [45], [46]. Based on these results, no evidence has been found for the variability in supraspinatus stabilizing function between individuals that has been proposed by some, based on EMG activity [13]. Moreover the stability ratio did not change with the type of movement in the SI direction but did change with movement type and elevation in the AP direction, where the different anatomical subregions of the muscle have been found to act differently [47]. However, the stability ratio of the supraspinatus muscle in this study was averaged across the multiple line elements that represented the different four regions of the supraspinatus muscle model [26]. It is therefore possible that different anatomical subregions of the supraspinatus could have even more distinct functions [47].

In the superficial shoulder muscles the stability ratios were influenced by the amount of elevation, with the exception of the deltoid in the AP direction, thus mostly confirming the hypothesis. Moreover, a significant movement main effect and interaction between elevation and movement were also found, with the exception of the pectoralis in the SI direction, contrary to the hypothesis that the plane of elevation, i.e. the type of movement, would not affect the ratios. The magnitude of the stability ratios of latissimus and pectoralis muscles is 

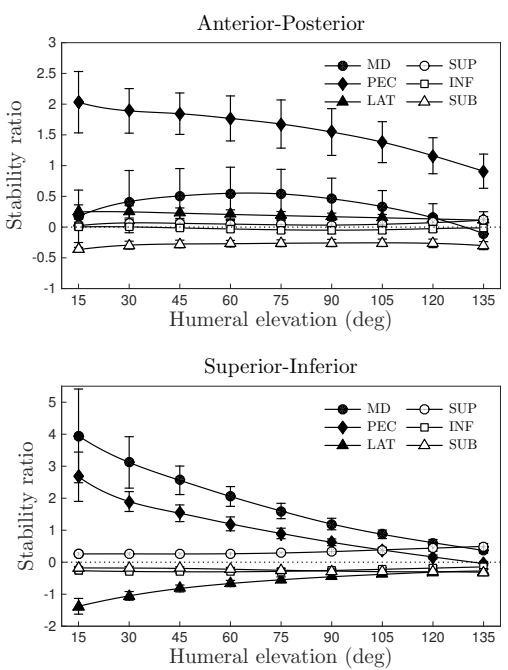

(a) Abduction
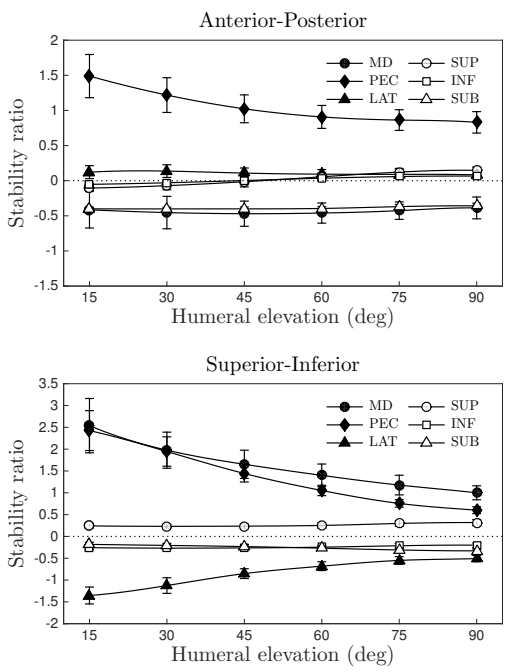

(d) Ipsilateral Reaching
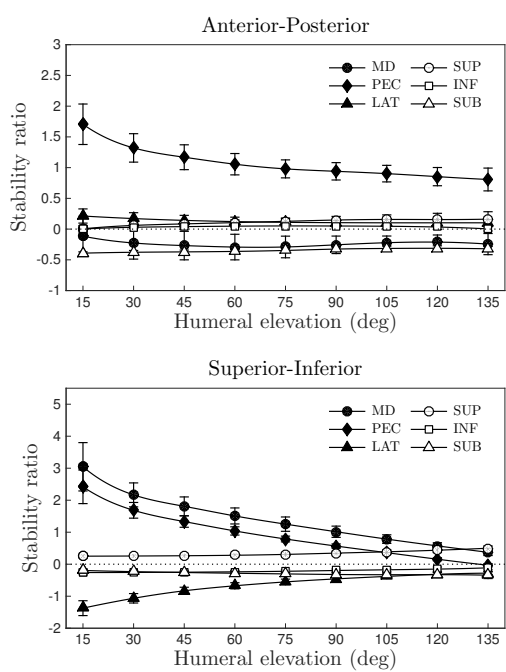

(b) Scapular Plane Elevation
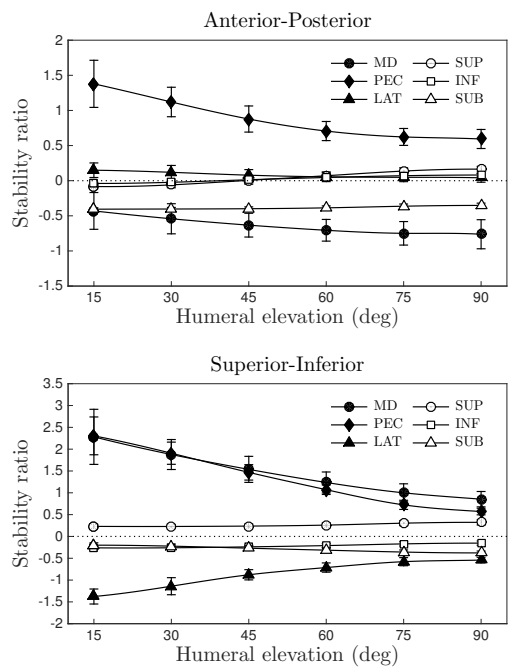

(e) Front Reaching

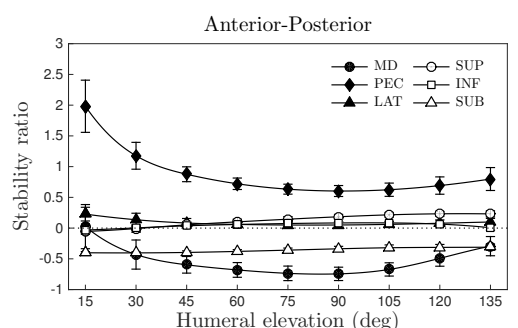

Superior-Inferior

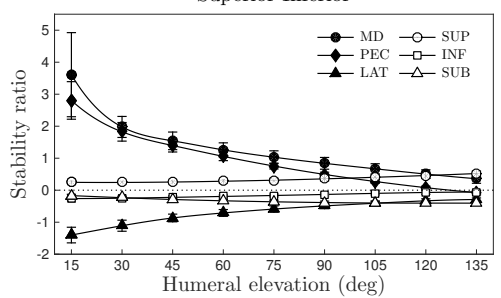

(c) Forward Flexion
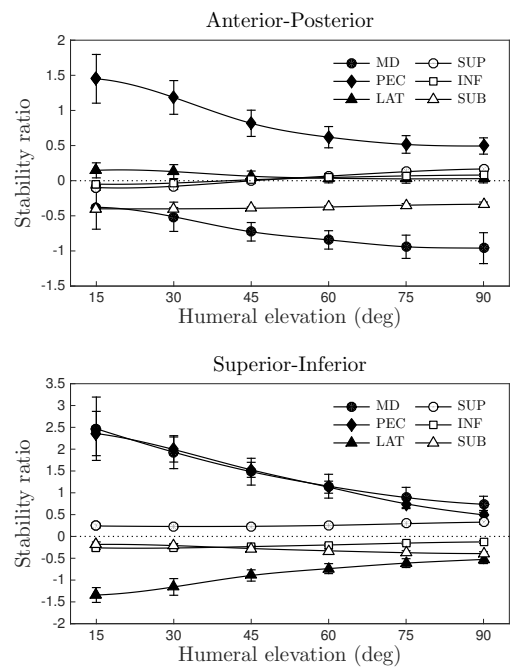

(f) Contralateral Reaching

Fig. 4. Stability ratio of each muscle in the anterior-posterior and superior-inferior direction in each of the measured movements, averaged across ten participants. A lower absolute stability ratio corresponds to a greater potential contribution of that muscle to glenohumeral joint stability. Open symbols represent rotator cuff muscles. Error bars indicate standard deviation. Muscles: MD: middle deltoid; PEC: clavicular part of pectoralis major; LAT: latissimus dorsi; SUP: supraspinatus; INF: infraspinatus; SUB: subscapularis.

in line with previous findings [18], as well as that of the deltoid [22]. In the superior direction, both the middle deltoid, pectoralis major and latissimus dorsi were characterized by a high destabilizing potential in the initial phase of elevation, which decreased as the arm was lifted (Figure 4). As the movements progressed and the humeral axis became more perpendicularly oriented to the glenoid cavity, the compressive force components increased and eventually exceeded the shear component. Although the middle deltoid and pectoralis are generally seen as joint mobilizers [19], [48], [13], the current research demonstrates that the role of these muscles can vary within and between movements. As can be observed by the sign of the stability ratio in the SI direction, the shear forces of the middle deltoid and pectoralis in the superior direction appear to be counteracted by the inferior shear force of the latissimus dorsi. On the basis of the contrasts the stability ratios in all the examined superficial muscles were additionally found to be influenced by the type of task and level of elevation in SI, AP direction or both, with greater stability ratios, and thus greater shear over compressive forces, at lower levels of elevations. This provides evidence that the stabilizing role of these superficial shoulder muscles varies with the level of elevation depending on the movement type.

\section{Limitations}

The musculoskeletal model used in this study was based on a single cadaver specimen. It cannot therefore be ruled out that individual morphological differences might have caused inaccuracies in the model estimations. In particular, glenoid version and inclination can vary between participants. The values of glenoid version and inclination used in the model are within the anatomical range of the adult population [49]. 
Changes in glenoid inclination within its anatomical range affect the stability ratio by approximately $\pm 10 \%$ [50], whereas increase in glenoid retroversion is associated with decreased inferior and posterior glenohumeral forces up to a maximum of $20 \%$ approximately [51].

The absence of a glenohumeral force constraint could have led to an overestimation of the shear over compressive forces, and thus higher stability ratios, particularly in the earlier phase of humeral elevation where the shear forces dominate. However the estimated resultant glenohumeral joint force was well within the range reported by other studies, and the stability ratios in abduction agreed with the literature from comparable models [22]. Concerning the validity of the stability ratio estimates above $90^{\circ}$, as mentioned above the calculations of stability ratio are based only on spatial properties of the model rather than dynamical muscle properties and are therefore independent of the magnitude of the estimated muscle force.

Finally, the musculoskeletal model used in this study did not include proprioceptive feedback. Afferent feedback from the mechanoreceptors in the rotator cuff muscles is thought to contribute to glenohumeral stability by eliciting synergistic reflex activity of the muscles [52]. Although the exact way in which rotator cuff afferent feedback functions in humans is still being investigated [53], [54], a comprehensive computational model should also include proprioceptive mechanisms.

\section{CONCLUSION}

Results of the current study confirm the functional role of the rotator cuff muscles as dynamic stabilizers during single plane elevation movements and reaching tasks. We showed that the functional role of the examined superficial shoulder muscles is mostly dependent on the amount of humeral elevation and the type of movement and should therefore always be considered in the context of the spatial characteristics of the movement. The findings are useful to predict situations and movements where the joint becomes more unstable due to greater shear over compressive forces. Further research should aim at applying the personalization technique for the scapulohumeral rhythm described in this study to populations with abnormal shoulder motion, such as scapular dyskinesia, to reveal how pathological adaptations can affect glenohumeral stability.

\section{REFERENCES}

[1] M. Peat, "Functional anatomy of the shoulder complex," Physical Therapy, vol. 66, no. 12, pp. 1855-65, 1986.

[2] S. Lippitt and F. Matsen, "Mechanisms of glenohumeral joint stability," Clinical Orthopaedics and Related Research, no. 291, pp. 20-8, 1993.

[3] P. Omoumi, P. Teixeira, F. Lecouvet, and C. B. Chung, "Glenohumeral joint instability," Journal of Magnetic Resonance Imaging, vol. 33, no. 1, pp. 2-16, 2011.

[4] M. Urwin, D. Symmons, T. Allison, T. Brammah, H. Busby, M. Roxby, A. Simmons, and G. Williams, "Estimating the burden of musculoskeletal disorders in the community: the comparative prevalence of symptoms at different anatomical sites, and the relation to social deprivation," Annals of The Rheumatic Diseases, vol. 57, no. 11, pp. 649-55, 1998.

[5] G. Johnson, N. Bogduk, A. Nowitzke, and D. House, "Anatomy and actions of the trapezius muscle," Clinical Biomechanics, vol. 9, pp. 4450, 1994.

[6] N. K. Poppen and P. S. Walker, "Normal and abnormal motion of the shoulder," The Journal of Bone and Joint Surgery. American Volume, vol. 58, no. 2, pp. 195-201, 1976.
[7] J. H. de Groot and R. Brand, "A three-dimensional regression model of the shoulder rhythm," Clinical Biomechanics, vol. 16, no. 9, pp. 735-43, 2001.

[8] E. Codman, The shoulder. Boston, USA: Thomas Todd Co, 1934.

[9] S. B. Lippitt, J. E. Vanderhooft, S. L. Harris, J. A. Sidles, D. T. Harryman, and F. A. Matsen, "Glenohumeral stability from concavitycompression: A quantitative analysis," Journal of Shoulder and Elbow Surgery, vol. 2, no. 1, pp. 27-35, 1993.

[10] S. R. Ward, E. R. Hentzen, L. H. Smallwood, R. K. Eastlack, K. A. Burns, D. C. Fithian, J. Friden, and R. L. Lieber, "Rotator cuff muscle architecture: implications for glenohumeral stability," Clinical Orthopaedics and Related Research, vol. 448, pp. 157-63, jul 2006.

[11] C. E. Boettcher, I. Cathers, and K. A. Ginn, "The role of shoulder muscles is task specific," Journal of Science and Medicine in Sport, vol. 13, no. 6, pp. 651-6, 2010.

[12] D. Wattanaprakornkul, I. Cathers, M. Halaki, and K. A. Ginn, "The rotator cuff muscles have a direction specific recruitment pattern during shoulder flexion and extension exercises," Journal of Science and Medicine in Sport, vol. 14, no. 5, pp. 376-382, 2011.

[13] P. B. De Witte, S. Werner, L. M. ter Braak, H. E. J. Veeger, R. G. H. H. Nelissen, and J. H. de Groot, "The Supraspinatus and the Deltoid - Not just two arm elevators," Human Movement Science, vol. 33, no. 1, 2014.

[14] M. Nordin and V. H. Frankel, Basic Biomechanics of the Musculoskeletal System. Baltimore, USA: Lippincott Williams \& Wilkins, 2001.

[15] H. E. J. Veeger and F. C. T. van der Helm, "Shoulder function: the perfect compromise between mobility and stability," Journal of Biomechanics, vol. 40, no. 10, pp. 2119-29, 2007.

[16] T. Kido, E. Itoi, S.-B. Lee, P. G. Neale, and K.-N. An, "Dynamic stabilizing function of the deltoid muscle in shoulders with anterior instability," American Journal of Sports Medicine, vol. 31, no. 3, pp. 399-403, 2003.

[17] S. B. Lee, K. J. Kim, S. W. O'Driscoll, B. F. Morrey, and K. N. An, "Dynamic glenohumeral stability provided by the rotator cuff muscles in the mid-range and end-range of motion. A study in cadavera," The Journal of Bone and Joint Surgery. American Volume, vol. 82, no. 6, pp. 849-57, 2000.

[18] D. C. Ackland and M. G. Pandy, "Lines of action and stabilizing potential of the shoulder musculature," Journal of Anatomy, vol. 215, no. 2, pp. 184-97, 2009.

[19] N. K. Poppen and P. S. Walker, "Forces at the glenohumeral joint in abduction," Clinical Orthopaedics and Related Research, no. 135, pp. $165-70,1978$.

[20] P. Favre, J. G. Snedeker, and C. Gerber, "Numerical modelling of the shoulder for clinical applications," Philosophical Transactions of the Royal Society of London A: Mathematical, Physical and Engineering Sciences, vol. 367, no. 1895, pp. 2095-2118, 2009.

[21] P. Favre, M. Senteler, J. Hipp, S. Scherrer, C. Gerber, and J. G. Snedeker, "An integrated model of active glenohumeral stability," Journal of Biomechanics, vol. 45, no. 13, pp. 2248-55, 2012.

[22] T. Yanagawa, C. J. Goodwin, K. B. Shelburne, J. E. Giphart, M. R. Torry, and M. G. Pandy, "Contributions of the individual muscles of the shoulder to glenohumeral joint stability during abduction," Journal of Biomechanical Engineering, vol. 130, no. 2, p. 021024, 2008.

[23] R. A. Ekstrom, G. L. Soderberg, and R. A. Donatelli, "Normalization procedures using maximum voluntary isometric contractions for the serratus anterior and trapezius muscles during surface EMG analysis," Journal of Electromyography and Kinesiology, vol. 15, no. 4, pp. 41828, 2005.

[24] A. Seth, M. Sherman, J. A. Reinbolt, and S. L. Delp, "OpenSim: a musculoskeletal modeling and simulation framework for in silico investigations and exchange," Procedia IUTAM, vol. 2, pp. 212-232, 2011.

[25] S. L. Delp, F. C. Anderson, A. S. Arnold, P. Loan, A. Habib, C. T. John, E. Guendelman, and D. G. Thelen, "OpenSim: Open-Source Software to Create and Analyze Dynamic Simulations of Movement," IEEE Transactions on Biomedical Engineering, vol. 54, no. 11, pp. 19401950, 2007.

[26] E. K. Chadwick, D. Blana, R. F. Kirsch, and A. J. van den Bogert, "Realtime simulation of three-dimensional shoulder girdle and arm dynamics," IEEE Transactions on Biomedical Engineering, vol. 61, no. 7, pp. 194756, 2014.

[27] A. A. Nikooyan, H. E. J. Veeger, E. K. J. Chadwick, M. Praagman, and F. C. T. van der Helm, "Development of a comprehensive musculoskeletal model of the shoulder and elbow," Medical \& Biological Engineering \& Computing, vol. 49, no. 12, pp. 1425-35, 2011. 
[28] J. Marchi, D. Blana, and E. K. Chadwick, "Glenohumeral stability during a hand-positioning task in previously injured shoulders," Medical \& Biological Engineering \& Computing, vol. 52, pp. 251-256, 2014.

[29] J. A. Prinold, A. F. Shaheen, and A. M. Bull, "Skin-fixed scapula trackers: A comparison of two dynamic methods across a range of calibration positions," Journal of Biomechanics, vol. 44, no. 10, pp. 2004-2007, 2011.

[30] N. Barnett, R. Duncan, and G. Johnson, "The measurement of three dimensional scapulohumeral kinematics a study of reliability," Clinical Biomechanics, vol. 14, no. 4, pp. 287-290, 1999.

[31] G. Wu, F. C. T. van der Helm, H. E. J. D. Veeger, M. Makhsous, P. Van Roy, C. Anglin, J. Nagels, A. R. Karduna, K. McQuade, X. Wang, F. W. Werner, and B. Buchholz, "ISB recommendation on definitions of joint coordinate systems of various joints for the reporting of human joint motion-Part II: shoulder, elbow, wrist and hand," Journal of Biomechanics, vol. 38, no. 5, pp. 981-992, 2005.

[32] X. Xu, C. R. Dickerson, J. H. Lin, and R. W. McGorry, "Evaluation of regression-based 3-d shoulder rhythms," Journal of Electromyography and Kinesiology, vol. 29, pp. 28-33, 2016.

[33] Y. Blache and M. Begon, "Influence of shoulder kinematic estimate on joint and muscle mechanics predicted by musculoskeletal model," IEEE Transactions on Biomedical Engineering, vol. 65, pp. 715-722, 2018.

[34] D. G. Thelen, F. C. Anderson, and S. L. Delp, "Generating dynamic simulations of movement using computed muscle control," Journal of Biomechanics, vol. 36, no. 3, pp. 321-328, 2003.

[35] D. Blana, J. G. Hincapie, E. K. Chadwick, and R. F. Kirsch, "A musculoskeletal model of the upper extremity for use in the development of neuroprosthetic systems," Journal of Biomechanics, vol. 41, no. 8, pp. 1714-21, 2008.

[36] M. Mirakhorlo, M. R. Azghani, and S. Kahrizi, "Validation of a musculoskeletal model of lifting and its application for biomechanical evaluation of lifting techniques," Journal of Research in Health Sciences, vol. 14, no. 1, pp. 23-8, 2014.

[37] A. Wibawa, N. Verdonschot, J. Halbertsma, J. Burgerhof, R. Diercks, and G. Verkerke, "Musculoskeletal modeling of human lower limb during normal walking, one-legged forward hopping and side jumping: Comparison of measured EMG and predicted muscle activity patterns," Journal of Biomechanics, vol. 49, no. 15, pp. 3660-3666, 2016.

[38] A. A. Nikooyan, H. E. J. Veeger, P. Westerhoff, F. Graichen, G. Bergmann, and F. C. T. van der Helm, "Validation of the Delft Shoulder and Elbow Model using in-vivo glenohumeral joint contact forces," Journal of Biomechanics, vol. 43, no. 15, pp. 3007-14, 2010.

[39] N. C. Silver and W. P. Dunlap, "Averaging correlation coefficients: Should Fisher's z transformation be used?," Journal of Applied Psychology, vol. 72, no. 1, pp. 146-148, 1987.

[40] E. Nelson-Wong, S. Howarth, D. A. Winter, and J. P. Callaghan, "Application of autocorrelation and cross-correlation analyses in human movement and rehabilitation research," Journal of Orthopaedic \& Sports Physical Therapy, vol. 39, no. 4, pp. 287-295, 2009.

[41] W. Wu, P. V. Lee, A. L. Bryant, M. Galea, and D. C. Ackland, "Subjectspecific musculoskeletal modeling in the evaluation of shoulder muscle and joint function," Journal of Biomechanics, vol. 49, no. 15, pp. 36263634, 2016.
[42] J. M. de Toledo, D. C. Ribeiro, M. P. de Castro, F. C. Forte, T. S. Körbes, M. W. Rusch, and J. F. Loss, "Comparison of shoulder resultant net moment between three different exercises and load conditions," Physiotherapy Theory and Practice, vol. 29, no. 2, pp. 124-32, 2013.

[43] S. Sangwan, R. A. Green, and N. F. Taylor, "Stabilizing characteristics of rotator cuff muscles: a systematic review," Disability and Rehabilitation, pp. 1-11, 2014.

[44] R. F. Escamilla, K. Yamashiro, L. Paulos, and J. R. Andrews, "Shoulder muscle activity and function in common shoulder rehabilitation exercises," Sports Medicine, vol. 39, no. 8, pp. 663-685, 2009.

[45] S. M. Howell, A. M. Imobersteg, D. H. Seger, and P. J. Marone, "Clarification of the role of the supraspinatus muscle in shoulder function," The Journal of Bone \& Joint Surgery, vol. 68, no. 3, 1986.

[46] N. Wuelker, M. Korell, and K. Thren, "Dynamic glenohumeral joint stability," Journal of Shoulder and Elbow Surgery, vol. 7, no. 1, pp. 4352, 1998.

[47] J. J. Gates, J. Gilliland, M. H. McGarry, M. C. Park, D. Acevedo, M. J. Fitzpatrick, and T. Q. Lee, "Influence of distinct anatomic subregions of the supraspinatus on humeral rotation," Journal of Orthopaedic Research, vol. 28, no. 1, pp. 12-17, 2010.

[48] D. C. Ackland, P. Pak, M. Richardson, and M. G. Pandy, "Moment arms of the muscles crossing the anatomical shoulder," Journal of Anatomy, vol. 213, no. 4, pp. 383-390, 2008.

[49] R. S. Churchill, J. J. Brems, and H. Kotschi, "Glenoid size, inclination, and version: An anatomic study," Journal of Shoulder and Elbow Surgery, vol. 10, no. 4, pp. 327-332, 2001.

[50] B. Moor, R. Kuster, G. Osterhoff, D. Baumgartner, C. Werner, M. Zumstein, and S. Bouaicha, "Inclination-dependent changes of the critical shoulder angle significantly influence superior glenohumeral joint stability," Clinical Biomechanics, vol. 32, pp. 268-273, 2016.

[51] T. A. Shapiro, M. H. McGarry, R. Gupta, Y. S. Lee, and T. Q. Lee, "Biomechanical effects of glenoid retroversion in total shoulder arthroplasty," Journal of Shoulder and Elbow Surgery, vol. 16, no. 3, Supplement, pp. S90-S95, 2007.

[52] L. P. Diederichsen, J. Nørregaard, P. Dyhre-Poulsen, A. Winther, G. Tufekovic, T. Bandholm, L. R. Rasmussen, and M. Krogsgaard, "The activity pattern of shoulder muscles in subjects with and without subacromial impingement," Journal of Electromyography and Kinesiology, vol. 19, no. 5, pp. 789-99, 2009.

[53] J. B. Myers, C. A. Wassinger, and S. M. Lephart, "Sensorimotor contribution to shoulder stability: Effect of injury and rehabilitation," Manual Therapy, vol. 11, no. 3, pp. 197-201, 2006.

[54] A. Day, N. F. Taylor, and R. A. Green, "The stabilizing role of the rotator cuff at the shoulder - responses to external perturbations," Clinical Biomechanics, vol. 27, no. 6, pp. 551-556, 2012. 